\title{
O.S.P.
}

L'orientation scolaire et professionnelle

$39 / 1 \mid 2010$

S'orienter : construire sa vie

\section{Le Life designing face aux aléas postmodernes du conseil en orientation}

Life designing and the postmodern vagaries of counselling

Jean-Pierre Boutinet et Christian Heslon

\section{OpenEdition}

\section{Journals}

Édition électronique

URL : http://journals.openedition.org/osp/2463

DOI : 10.4000/osp.2463

ISSN : 2104-3795

Éditeur

Institut national d'étude du travail et d'orientation professionnelle (INETOP)

Édition imprimée

Date de publication : 15 mars 2010

Pagination : 53-71

ISSN : 0249-6739

\section{Référence électronique}

Jean-Pierre Boutinet et Christian Heslon, « Le Life designing face aux aléas postmodernes du conseil en orientation », L'orientation scolaire et professionnelle [En ligne], 39/1 | 2010, mis en ligne le 15 mars 2013, consulté le 16 décembre 2020. URL : http://journals.openedition.org/osp/2463 ; DOI : https:// doi.org/10.4000/osp.2463

Ce document a été généré automatiquement le 16 décembre 2020.

(c) Tous droits réservés 


\title{
Le Life designing face aux aléas postmodernes du conseil en orientation
}

\author{
Life designing and the postmodern vagaries of counselling
}

Jean-Pierre Boutinet et Christian Heslon

\section{Introduction}

1 Le présent papier entend porter un point de vue critique sur la contribution originale intitulée "Life designing: A paradigm for career construction in the $21^{\text {st }}$ century ». Cette contribution cherche à construire un nouveau modèle de l'orientation à partir du paradigme du life designing. Issue d'une collaboration internationale, principalement européenne, elle est co-écrite par neuf rédacteurs originaires de six pays européens et des États-Unis. En ce début de $21^{\mathrm{e}}$ siècle, ces co-auteurs partent du constat qu'une nouvelle organisation sociale du travail génère chez l'individu, en lieu et place des anciennes trajectoires linéaires de la modernité, de nouveaux parcours professionnels plus aléatoires, aux devenirs heurtés et fluctuants qui caractérisent nos existences contemporaines - autant faites de bifurcations inattendues que d'opportunités insaisissables. De tels parcours sont tout à fait caractéristiques des environnements postmodernes. Une telle mutation dans l'orientation de la trajectoire au parcours implique de penser d'autres modes d'orientation professionnelle plus adéquats. Les coauteurs se focalisent alors sur un modèle qu'ils ébauchent et considèrent comme transversal de leurs cultures d'appartenance. Ils l'intitulent Life-designing model, traduit en français par l'expression «construire sa vie ». Il s'agit, selon ces rédacteurs, d'un «modèle » associé à des «méthodes d'accompagnement des personnes» (a counseling model and methods) destiné à profiler des «interventions d'accompagnement en orientation" (career intervention). Se mettre d'accord sur un modèle unique entre professionnels de différents pays à l'ère de la mondialisation du conseil en orientation évite, selon ces co-auteurs, de devoir adapter par la suite un modèle conçu dans un pays 
donné, pour pouvoir l'exporter dans un autre. Cette modélisation assoit sa légitimité en s'appuyant sur cinq présuppositions que nous rappellerons plus loin, ladite modélisation se réclamant épistémologiquement du constructionnisme social, lequel privilégie au sein du conseil en orientation les interactions sociales entre la personne qui s'oriente et le professionnel qui est censé l'aider à s'orienter par elle-même, via la médiation du discours. De telles interactions sont finalisées par la construction de soi et de son parcours dans une perspective holiste et préventive.

2 Nous rappellerons dans un premier temps les présupposés sur lesquels s'appuient les co-auteurs pour justifier un tel modèle, ainsi que la configuration de ce dernier, pour organiser ensuite notre regard critique autour de cinq interrogations :

- Dans quelle mesure la mondialisation prétendument facilitatrice ainsi actée s'affranchit-elle véritablement des ancrages culturels et linguistiques des co-auteurs ici réunis autour de l'idéal d'un Life-designing espérantiste?

5 - À propos de l'orientation dont il est ici question, de quel sujet adulte en devenir traite-t-on et à quel sujet s'adresse-t-on?

6 - Derrière le modèle constructionniste déployé, quelle est la conception de l'orientation sous-tendue par ce modèle et à quelle actualité prétend-elle répondre?

7 - Quant au professionnel du conseil en orientation, que devient-il dans tout cela?

\section{Le modèle du Life designing appréhendé par ses présupposés fondateurs}

Rappelons tout d'abord que ce modèle du Life designing tel qu'explicité par ses neuf auteurs se fonde sur les cinq présupposés suivants :

- Redonner toute son importance au contexte, celui de la situation d'orientation, de préférence à la recherche de traits universalisables comme ce fut souvent le cas antérieurement, notamment avec les traits de personnalité ou les facteurs révélateurs de capacités stables. Cette importance conférée au contexte vise à remettre en cause la vieille attitude adéquationniste qui cherchait à diagnostiquer la meilleure correspondance possible entre la personne telle qu'elle se perçoit et l'environnement socioprofessionnel potentiel dans lequel elle évolue.

10 - Privilégier le processus sur la prescription en se focalisant sur les stratégies de survie et de coping de la personne, là où le conseiller d'orientation continue encore à raisonner en termes de trajectoire ou de carrière, c'est-à-dire en termes de choix unique plutôt que d'alternatives plurielles autorisant des changements fréquents d'orientation du fait très actuel d'une surcharge d'informations et d'exigences professionnelles sans cesse en mutations.

11 - Adopter des stratégies itératives dans le temps, compte tenu des dynamiques d'orientation qui ont cessé d'obéir à une causalité linéaire pour devenir principalement non linéaires, ce qui empêche des prédictions sur l'avenir plus ou moins immédiat ; ces stratégies se développent dans des situations d'interaction non rationnelles, c'est-àdire en partie imprévisibles; elles entendent donc ouvrir sur un travail de coconstruction et sur un mode d'anticipation préventive.

L'orientation scolaire et professionnelle, 39/1 | 2010 
12 - Se centrer sur les processus interminables de construction et de reconstruction subjectives des parcours à partir du récit qu'en font les personnes en orientation ; car les parcours de vie entre famille, travail et formation sont devenus pluriels et amènent à une diversité grandissante de réalités individuelles, générant des identités ellesmêmes plurielles; l'encadrement de ces parcours ne saurait donc être assuré par des conseillers d'orientation formés la plupart du temps aux méthodes scientifiques standardisées et normées.

13 - Procéder empiriquement à une évaluation pluridimensionnelle du conseil en orientation à partir d'une configuration stable de variables bien identifiées, plutôt que de pratiquer des évaluations unidimensionnelles en ne tenant compte que d'une seule variable.

14 C'est en s'appuyant sur ces cinq présupposés qu'a été élaboré le modèle du Life designing qui comporte quatre caractéristiques essentielles : il s'étend aux différents âges de la vie (considérée comme un parcours non prédictible et faite d'un processus individuel influencé par des facteurs environnementaux devant amener à la construction de soi) ; il se veut holistique (incluant les différents rôles tenus par la personne au-delà du seul parcours professionnel et permettant de travailler autant sur la construction de soi que sur celle du parcours) ; par ailleurs il se présente comme contextuel (car soucieux de valoriser la variété des environnements fréquentés par la personne et qui font sens pour elle); enfin, il cherche à être plus préventif que prédictif (faisant porter son attention sur l'avenir de la personne, sur ses possibilités effectives de choix pour qu'elles en soient accrues tout en portant une attention particulière aux situations à risque). En prenant pour cadre ces quatre caractéristiques, le modèle poursuit trois visées dans les interventions d'accompagnement à la construction de sa vie : accroître l'adaptabilité de la personne pour anticiper les changements, développer sa narrabilité entrevue comme capacité à raconter son parcours de vie, affermir son intentionnalité à s'engager dans des activités effectives qui font sens. Si l'adaptabilité est relative au changement à gérer, la narrativité renvoie au contraire à la continuité à assumer ; c'est une telle relation paradoxale, formulée en termes de flexibilité-fidélité, qui permet à la personne de structurer l'intention d'entreprendre des activités qui font sens pour elle.

15 Le modèle d'intervention pour accompagner la construction de sa vie dans son déroulement chronologique va alors pouvoir s'organiser en six étapes :

16 - bénéficiaire et conseiller circonscrivent le problème d'orientation, son contexte et identifient les attentes de part et d'autre, définissent les objectifs de leur interaction visant à encourager le bénéficiaire à raconter l'histoire de ses difficultés à s'orienter ;

17 - après l'identification du problème, le conseiller aide le consultant à structurer ses expériences et ses espérances, ses relations aux autres, ses anticipations dans un récit d'exploration de soi ;

18 - il s'agit ensuite d'ouvrir des perspectives en transformant l'implicite du récit en explicite plus objectif - dans une mise à distance des expériences et espérances, plus claire, plus consistante et aussi plus critique ;

19 - l'interaction facilite alors un repositionnement du problème initial à travers les perspectives ainsi esquissées, conduisant à la formulation de nouvelles anticipations ;

20 - le bénéficiaire dessine un plan d'engagement dans de nouvelles expériences, en s'assurant de l'appui d'un certain nombre d'acteurs ressources ;

21 - suivi sur le court et le long terme. 

pour les co-auteurs, de former les conseillers, praticiens à l'accompagnement d'orientation, comme il est indispensable de lancer des programmes de recherche, notamment sur les processus sous-jacents aux interventions d'accompagnement.

\section{Pertinence des fondamentaux sur lesquels s'appuie le modèle proposé}

Passons en revue les présupposés qui entendent légitimer le modèle « Life designing for career construction in the $21^{\text {st }}$ century " formalisé autour du conseil d'orientation. D'emblée, reconnaissons qu'un tel modèle a le mérite de regrouper les éléments essentiels à prendre en compte pour repenser aujourd'hui l'orientation, éléments sur lesquels il y a généralement accord entre partenaires chercheurs et professionnels de l'orientation - même si cet accord n'est pas toujours suivi de passage à l'acte ; nous ne faisons ici que les rappeler.

L'une des données essentielles sur lesquelles s'appuie le modèle est la nécessité de contextualiser l'orientation au regard de l'environnement dans lequel elle se déploie ; cet environnement est fait, entre autres, d'une grande mobilité individuelle couplée de flexibilité organisationnelle et d'un allongement spectaculaire de la durée de vie des personnes qui s'orientent. Ces deux éléments mis ensemble nous imposent désormais de penser l'orientation aux différents âges de la vie et non plus de la réserver aux seuls jeunes scolarisés, comme on l'a fait pendant quatre-vingts ans. Il s'agit par ailleurs de donner l'avantage au processus du parcours dans ses incessants remaniements sur les prescriptions qui figent, de valoriser les stratégies itératives et, pour ce faire, de recourir à une mise en langage de son expérience par le bénéficiaire lui-même au travers d'une double itérativité : d'une part, celle d'un va-et-vient entre ce qu'il a réalisé et la façon par laquelle il le met à distance par les mots; d'autre part, celle d'un second va-et-vient entre ce qu'il vient de verbaliser et l'intention reformulée qu'il projette sur son avenir à réaménager.

Autre point fort souligné par le modèle et qui renvoie à un signe des temps actuels : l'importance dévolue au pluriel dans le processus d'orientation. Il s'agit de penser une orientation plurielle et non plus univoque, une orientation qui se fait, se défait, se recompose, bifurque, amenant à des choix simultanés et successifs. Cette orientation, dans sa pluralité, est à associer aussi à la diversité tant des parcours familial, de travail, professionnel, social que des identités personnelle, conjugale, familiale, professionnelle, sociale qui se structurent, se délitent ou se recomposent par lesdits parcours. Le pluriel renvoie aussi à la diversité des environnements, comme à la multiplicité des informations qui les traversent, qui sont à sélectionner, à hiérarchiser, à éliminer. Enfin, le pluriel touche l'évaluation des démarches d'orientation, tant dans la fonction conseil proprement dite, conduite par le professionnel, que dans celle du parcours d'orientation du bénéficiaire ; cette évaluation gagne désormais à recourir à une démarche pluridimensionnelle tant du conseil effectué que du parcours d'orientation déjà réalisé et projeté. Par évaluation pluridimensionnelle, il faut entendre la combinaison de plusieurs évaluations à mener simultanément à l'issue d'une séquence d'orientation : celle bien entendu des objectifs atteints au regard des objectifs fixés initialement, mais aussi celle des réussites concrétisées vis-à-vis des échecs vécus, comme celle des avantages acquis par rapport aux coûts consentis, celle

L'orientation scolaire et professionnelle, 39/1 | 2010 
de l'imprévu survenu en cours de route au regard du prévu anticipé, celle des résultats momentanément obtenus, à situer en regard de la situation originelle de départ, sans oublier la satisfaction que le bénéficiaire tire de la situation actuelle en comparaison avec son possible désarroi.

Enfin, en contraste avec le constructivisme cognitif moderne tel qu'il fut promu par Piaget (1967) à travers les autorégulations équilibrantes d'assimilation et d'accommodations chez le sujet, le modèle qui retient ici notre attention se recommande du constructionnisme social et s'inscrit délibérément dans un espace postmoderne (Gergen, 1991), centré non plus sur l'individualité mais sur la relation. Ce modèle fait implicitement référence aux travaux initiés par Gergen (2001) qui érige le concept de constructionism en véritable paradigme. Un tel paradigme entend se situer dans le prolongement mais en contrepoint du déconstructionnisme postmoderne, tel que Derrida (1967) l'a développé pour affronter l'état d'incertitude et de chaos dans lesquels se trouvait précipitée la pensée moderne. La déconstruction, si désespérée soit-elle, signe l'avènement de transformations majeures dans la conception que nous nous faisons, chacun, de nous-mêmes à l'ère du World Wide Web. Nous ne pouvons plus nous enfermer dans le solipsisme d'une subjectivité autarcique pas plus que nous ne pouvons prétendre nous appuyer sur le réalisme d'une vérité intemporelle alors que le monde se métamorphose sans cesse sous nos yeux. Dans un tel espace, sans repères, tenté par l'absurde, seule prime, nous indique Gergen, la recherche de sens ; or celle-ci nous est donnée par la relation : le sens naît de la relation. Il s'agit donc désormais de penser un être relationnel en communication tant avec le monde social qu'avec le monde non social, structuré autour d'une technologie de plus en plus invasive. Seule cette relation est porteuse de perspective d'avenir et constitue une manière plus viable de vivre ensemble la construction de cet avenir.

\section{La mondialisation facilitatrice peut-elle supprimer les ancrages culturels et linguistiques des co-auteurs?}

La validité interculturelle invoquée par les co-auteurs pour appuyer la légitimité du modèle proposé est un point fort du travail d'échange et de concertation entre des chercheurs issus de différents pays et, en même temps, il constitue un point faible. En effet, l'aspect positif est que le modèle manifeste des convergences interculturelles intéressantes quant aux carences actuelles des différents systèmes d'orientation face à une société postmoderne de la mobilité et de la flexibilité qui a relégué au magasin des accessoires la relative stabilité d'antan. Il témoigne par le fait même de l'urgence à devoir repenser collectivement nos modes d'orientation dans les espaces postmodernes actuels par la mise en place de nouvelles formes de remédiation. Mais, et c'est l'aspect négatif, le modèle fait bon marché de la mondialisation qu'il évoque comme opportunité, sans prendre suffisamment en compte, semble-t-il, le fait que cette mondialisation rendue facile au sens que lui donne Milner (2006), notamment par la circulation de l'information et la communication, ne saurait abolir les différences culturelles. Privilégier l'interculturel sans trop de précaution constitue ici un acquis problématique à un double point de vue. D'abord, les propositions faites n'échappent pas dans leur formulation à des expressions très, voire trop, générales, relatives à "l'adaptabilité de la personne », aux " processus sous-jacents aux interventions ", à la « diversité des informations disponibles, aux changements effectifs de société »... Il y a 
là, par nécessité interculturelle, une sorte de nivellement indifférencié, une mise entre parenthèses des particularismes culturels propres à chaque système national, ce qui tend à appauvrir le modèle ; la prise en compte de ces particularismes aurait permis de proposer des variantes. Ainsi, la particularité française de considérer l'orientation au niveau scolaire dans son autarcie par rapport aux réorientations liées aux autres âges de la vie pose des problèmes de professionnalité du conseil tout à fait spécifiques à prendre en compte dans le cadre d'une scolarité prolongée, encore trop peu articulée sur les mobilités adultes ultérieures.

Ensuite si nous nous déplaçons de l'interculturel vers son voisin, l'interlinguistique, à travers la référence à l'anglais utilisé ici comme langue véhiculaire commune, nous constatons vite que les concepts utilisés au sein du modèle explicité dans cette langue ne sauraient prétendre à une forme de mondialisation sans réserve lorsqu'ils sont traduits dans une autre langue, si cette autre langue n'en respecte pas ce qui fait leur spécificité anglo-saxonne; trois exemples entre autres viennent à l'appui de cette réserve, qui gênent la compréhension que l'on peut se faire de la traduction française. Mais sans traduction, le problème demeure entier, chaque lecteur interprétant alors à sa façon dans sa grille culturelle les concepts employés.

Ainsi le paradigme central du modèle, le designing est rendu en français par construction, lequel terme dans la traduction française occupe une place écrasante car en plus de designing, il traduit en même temps building, construction. Or le designing se rapproche plus de la création que de la construction, comme sous-tendant toute activité humaine (Papanek, 1972), une création dynamisée par ses deux temps d'esquisse et de mise à l'épreuve, ce qui lui confère cette propriété paradoxale bien mise en valeur historiquement par Ciaravino (2004) d'être itérative, propriété signalée très opportunément d'ailleurs par le modèle. Mais si une création peut être itérative lorsqu'elle s'inspire du modèle du design, on ne peut pas dire d'une construction qu'elle est itérative : cela n'a pas de sens. La construction dans sa linéarité relève au contraire d'un processus peu, voire non itératif, si, du moins ici, comme d'ailleurs dans le modèle lorsqu'il évoque le building, on prend le concept de construction dans son sens opératoire et non dans un sens théorique lié à l'une ou l'autre forme de constructionnisme. Second exemple visant un autre concept très présent dans la version française, celui d'accompagnement, promu aujourd'hui au rang de vulgate dans notre culture française et même francophone mais intraduisible en anglais. Ceci fait que dans la version française, de par sa fréquence d'usage, l'accompagnement est élevé au rang de véritable paradigme alors que ses divers correspondants dans le texte anglais, en tant qu'équivalents plus lointains dans leurs variétés, restent plus discrets et éclatés, renvoyant à d'autres formes de professionnalité : counseling, help, intervention, guidance... On pourrait faire la même remarque pour le concept d'orientation, encore doublement marqué sémantiquement en français par sa liaison à un contexte particulier, l'école et à une classe d'âge déterminée, la jeunesse, alors qu'il correspond ici dans le modèle à des variantes anglaises comme vocation, career counseling, career intervention, career domain. Certes, on pourrait être tenté d'identifier orientation à career mais career ne saurait être réduit à la seule "orientation" puisqu'il traduit aussi " parcours». Toujours est-il que ces variantes, toutes rendues en traduction française par le même terme " orientation » ne comportent pourtant pas les ancrages historiques de ce dernier, liés au jeune âge et au scolaire, mais le lecteur français a-t-il les moyens de faire l'opération appropriée de décodage culturel ! 
30 La forme d'interculturalité ici développée risque donc d'aller à l'encontre du premier présupposé sur lequel s'appuie le modèle et qui est exprimé par le souci de vouloir contextualiser l'orientation en évitant de recourir à des traits universalisables. Qu'en est-il dans les autres langues des co-auteurs, en italien, en portugais, en néerlandais ?

\section{Entre modernité et postmodernité, de quelle orientation parlons-nous ici ?}

31 L'orientation s'est déclinée pendant plus d'un demi-siècle dans nos sociétés modernes, des années 1910 aux années 1960, selon deux modes privilégiés complémentaires :

- Le mode de l'orientation scolaire et professionnelle voit le jour à partir des premiers travaux de l'américain Parsons (1909) pour aménager l'insertion professionnelle des jeunes scolarisés qu'il s'agissait déjà d'adapter au marché du travail. Alors que Ginzberg (1951) dans son célèbre ouvrage sur la construction du choix professionnel ne visait encore que la jeunesse, c'est brusquement à partir de 1957 que Super va substituer la carrière à la profession pour étendre cette construction de carrière au-delà de la jeunesse aux différents âges de la vie adulte. Ainsi la pensée anglo-saxonne va-t-elle s'affranchir de cet ancrage de l'orientation aux confins de l'institution scolaire, une orientation propre aux jeunes pour envisager désormais la vocation et la career aux différents âges de la vie ; un tel affranchissement reste encore problématique au niveau français.

33 - Le mode de l'orientation activo-passif s'est imposé durant la même cinquantaine d'années, un mode dans lequel le conseiller orientait, alors que le jeune était orienté par l'une ou l'autre forme de conseil et d'intervention (Drevillon, 1966). Or depuis les années 1970-80, le changement culturel ambiant aidant, le jeune et le moins jeune sont mis au centre du dispositif d'orientation à travers l'ébauche de leur projet et, de ce fait, l'orientation a cessé en principe d'être déclinée sur le seul mode activo-passif pour l'être désormais sous la forme pronominale, typique de nos espaces post-modernes : le jeune n'est plus orienté, il s'oriente (Dubet, 1973); certes, cette injonction a souvent valeur de dénégation notamment lorsque, tout du moins en France, le conseil de classe continue à orienter en fonction des résultats scolaires. Malgré tout, le fait d'avoir un projet, ou ce qui en tient lieu, devient le sésame indispensable d'accès aux études et à la profession.

34 Aussi, lorsque dans les années 2000, on cherche à construire un nouveau modèle de l'orientation, on ne sait plus très bien, comme c'est le cas ici, par rapport à quel modèle de référence on se situe, un modèle qui met en scène le jeune ou l'adulte, l'orientation activo-passive ou l'orientation pronominale? Si dans l'exposé du modèle Life designing en ses débuts de formalisation, il est question du worker, de l'individual, du client pour désigner le bénéficiaire de l'orientation, donc l'adulte, jeune ou avancé en âge, en fin de modélisation, au niveau des préconisations, reviennent les pesanteurs historiques avec les children, parents, students, teachers. De même, la forme pronominale de l'orientation est bien exprimée dans la dénomination du modèle Life designing comme dans le cours du texte à travers certaines formulations du type project of life building mais pour être aussitôt encadrée par des «objectifs des interventions d'accompagnement à la construction de la vie » à travers les career interventions ou les vocational interventions érigées en modèle d'intervention. Alors que le modèle vise à faciliter la construction de 
sa vie par le bénéficiaire, il n'est question dans son déploiement que d'encadrement du Life designing par des vocational interventions: nous assistons là à une rencontre frontale entre une logique du «je» et une logique du «tu», elle-même au service d'une troisième logique plus impersonnelle, celle du «il», le modèle. De cette double confusion, jeune scolaire/adulte, forme activo-passive/forme pronominale, ne serait-il pas nécessaire d'en sortir à travers la nécessité de mettre en place une double distinction :

- À propos des bénéficiaires de l'orientation, jeunes et adultes, il devient indispensable de bien spécifier ce qui a trait à une primo orientation par rapport à une réorientation. La primo-orientation est échafaudée par un jeune, la plupart du temps scolarisé, seul, avec ses rêves d'enfance, ses premières expériences, son environnement familial et scolaire plus ou moins pesant sur ses choix et la façon par laquelle ce jeune met à l'épreuve des faits cette primo-orientation, en étant attentif à ces faits qui avec lui vont valider, confirmer, infirmer une telle orientation; cette primo-orientation, dans sa logique propre, est à distinguer d'une réorientation survenant ultérieurement, voire rapidement après une primo-orientation, d'autant plus rapidement sans doute que nous sommes dans une société de la communication et de la mobilité; alors que la primo-orientation exprime une continuité biographique, la réorientation opère un déplacement ; elle introduit une discontinuité, voire une rupture par rapport aux choix antérieurs; cette discontinuité ou rupture est à aménager en termes d'incertitude, de deuils, de crises, de transitions...

- De la même façon que l'on qualifie aujourd'hui les apprentissages tout au long de la vie (Carré, 2005) dans leurs dimensions formelle, non formelle et informelle, il devient opportun de distinguer ce qui a trait, d'une part, à l'orientation non formelle et informelle et, d'autre part, à l'orientation formelle : la première est une orientation plutôt pronominale, se déroulant certes bien ou mal, mais en l'absence de tout conseiller; la seconde s'apparente à une orientation activo-passive dans son formalisme - schématiquement le conseiller oriente, le jeune ou l'adulte est orienté - et se décline avec l'aide d'un professionnel de l'orientation, ce dernier ne pouvant toutefois exercer ses compétences que s'il a d'abord acquis une bonne connaissance du monde de l'orientation non formelle et informelle. L'orientation non formelle en ce qui la concerne est celle qui se fait au gré des expériences et rencontres rendues significatives par la personne qui s'oriente par elle-même, mais sans recours à un professionnel de l'orientation. L'orientation informelle quant à elle va se trouver initiée par le surgissement au sein d'un parcours de vie d'un évènement qui dans son imprévisibilité opère une rupture, déstructure des choix, remet en cause des perspectives et réorganise sur un nouveau mode l'horizon temporel à venir.

37 Le modèle proposé à notre critique amalgame trop souvent primo-orientation et réorientation sans donner suffisamment de consistance à l'une et à l'autre, sans les dialectiser. Car de la primo-orientation post-adolescente, issue d'un aménagement de l'imaginaire enfantin, il reste toujours quelque chose dans les réorientations ultérieures, si tant est que nos projets adultes sont toujours revisités par nos rêves d'enfants, ou mieux, constituent une façon de revisiter nos rêves enfantins. De même, le modèle ne distingue pas suffisamment orientation informelle et non formelle d'un côté, liées à une pronominalisation de l'orientation, et orientation formelle de l'autre, qui fait appel à l'étayage du conseil d'orientation lorsque les autres étayages environnementaux n'ont pas été suffisamment structurants. On devrait alors 
considérer cet étayage professionnel comme une situation limite et faire du conseil d'orientation un auxiliaire d'autant plus précieux qu'il n'est pas indispensable. Car penser l'orientation, c'est d'abord penser ses dimensions non formelles et informelles au cours desquelles se structure une vocation, une career, pour mieux comprendre de quels étayages doit se munir une orientation formelle, destinée à combler un déficit. De ce point de vue, si les apprentissages tout au long de la vie ne sauraient se passer de formalisme pour être menés à bon port, il en est tout autrement pour l'orientation qui gagne d'abord à rester non formelle ou informelle pour être bien conduite, l'orientation formelle ne survenant qu'en situation de manque et de vulnérabilité. C'est là faire du conseiller d'orientation un praticien à géométrie variable, plus éducateur dans le préventif, plus thérapeute dans le curatif. Privilégier le préventif c'est valoriser une éducation à l'orientation non formelle et informelle en encourageant une personne à s'orienter elle-même dans son autonomie. Rester dans le curatif, c'est toujours risquer d'instaurer des dépendances en revenant aux formes activo-passives de l'orientation. Mais ce risque doit être assumé lorsque les situations de déshérence l'exigent.

\section{Derrière le modèle constructionniste déployé, quelle conception de l'orientation se trouve en jeu ?} D'un côté, dans son optimisme préventif, il rappelle le modèle de l'adulte en perspective - illustré par le vocational develoment (Colarusso, Nemiroff, 1981) propre aux Trente Glorieuses de notre modernité tardive - un adulte ouvert aux possibilités offertes par les contextes, engagé dans des processus dynamiques, débouchant sur une progression avec des perspectives multiples. Or, face à une conjoncture déprimée et chaotique qui se traîne depuis plusieurs décennies, ce modèle optimiste de l'adulte en maturation est bien passé d'actualité. Mais d'un autre côté, cet adulte, pour se construire à travers son orientation, semble dans le modèle proposé ne pas pouvoir se passer de l'intervention d'un tiers, d'un conseiller, ce qui présuppose sa vulnérabilité, sa fragilité, voire même sa dépendance. Ce modèle socio-constructionniste semble de fait difficilement compatible avec l'ère du temps: l'adulte qui s'oriente ou qui est à orienter se trouve bien souvent confronté à sa propre précarité. À travers l'inextricable complexité de ses parcours, cet adulte se fait et se défait; dans les vicissitudes familiales et professionnelles, dans la privation d'emploi, dans l'accident de santé survenant à un moment ou à un autre de sa longévité, dans ses engagements multiples qui, par stress interposé, le menacent de fragmentation. Avec ou sans conseiller, l'adulte découvre vite en contexte de postmodernité, le caractère problématique, voire absurde de son parcours de vie qui n'est plus polarisé par un horizon à moyen ou long terme entrevu à travers quelques repères incontournables; d'où le recours, semble-t-il plus réaliste, à une orientation court-termiste d'un adulte pluriel à travers l'aménagement de transitions structurantes (Baubion-Broye, 1998) pour s'adapter aux incertitudes de l'existence (Bridges, 2006), transitions curieusement très peu évoquées par le présent modèle.

Ce modèle constructionniste utilise pour se nommer un concept emblématique, celui de designing. Sur le plan méthodologique certes, ce concept est le bien venu dans la mesure où il présuppose la mise en place de formes d'itérativité, proches du transitoire pour 
mener à bien son orientation; ces formes d'itérativité, ce sont celles par lesquelles se gèrent les projets d'orientation dans leur mode de détermination flou, ce qui évite d'assimiler l'orientation à des modes de détermination plus rigides - comme ceux de la décision et du choix qui excluent justement toute forme d'itérativité entre le travail de conception et celui de mise en œuvre. Mais au-delà de son intérêt méthodologique, ce concept de design incluant la forme substantivée designing s'est quelque peu affadi à l'ère postmoderne car, comme le souligne Fayolle (1998), tout devient design; le design est partout, nous le vivons au quotidien dans l'art de façonner et à force de tout dire, il ne veut plus rien dire. De plus, il garde un vieil arrière-fond théorique ambigu, laissant planer quelque ombre inquiétante pour penser l'orientation. En effet, celle-ci est susceptible par le design de s'apparenter à une forme de construction pouvant relever de l'auto-engendrement, avec ou sans conseiller. Car le design est un concept démiurgique, les théologiens anglais, dès le Moyen Âge, y avaient recours pour attribuer à Dieu cette propriété de pouvoir se créer par lui-même à travers ce que les théologiens ont appelé l'argument from design; il n'est donc pas étonnant que les gens de l'art, dans les arts majeurs de l'architecture, de la sculpture et de la peinture à la Renaissance italienne du Quattrocento aient eu recours au disegno pour légitimer leur capacité créative, entrevue comme démiurgique (Vasari, 1550). Encore aujourd'hui, les courants créationnistes, dans leur contestation des modèles évolutionnistes issus de la mouvance de Darwin utilisent l'argument from design pour signifier l'origine divine du monde (Bowman, 1979).

\section{Et le professionnel du conseil en orientation dans ce contexte, que devient-il ?}

40 Si on centre la démarche d'orientation sur le bénéficiaire, comme l'annonce le Life designing on ne saurait viser en même temps le conseiller d'orientation. Prendre au contraire comme focus le conseiller d'orientation et les exigences de sa nouvelle professionnalité implique de se décentrer par rapport au bénéficiaire. Or, le modèle soumis à notre investigation, sans afficher de parti pris, vogue inlassablement entre le bénéficiaire et le conseiller, ce qui place le lecteur en continuelle position de déséquilibre. Quoi qu'il en soit, dans plus d'un pays post-industriel, la fonction de conseiller d'orientation est entrée aujourd'hui en crise profonde en se laissant enfermer dans un cadre institutionnel, scolaire entre autres, qui, en lui donnant un statut pour sa reconnaissance, le rend prisonnier. Tributaire des espaces modernes au sein desquels il a été défini, le conseil d'orientation n'a pas pu ou su se redéfinir à l'ère postmoderne des réorientations, une ère marquée par le pluriel des références et des engagements, la complexité, la flexibilité des environnements, la mobilité des parcours et l'imprévisibilité des horizons temporels à venir, c'est-à-dire une ère qui n'a plus grand-chose à voir avec la relative stabilité moderne.

41 À la lumière de ce qui précède, nous voyons mieux en quoi le conseiller d'orientation gagne à devenir un généraliste du conseil dans les parcours de vie interinstitutionnels aux différents âges de l'existence ; ainsi, pouvoir confronter la primo-orientation aux réorientations ultérieures donne un champ de profondeur et une pertinence qui permettent de relativiser et d'enrichir les perspectives vocationnelles. De ce point de vue, le conseiller d'orientation a tout à gagner à délaisser la centralité des institutions pour se réfugier à leur périphérie; paradoxalement il en acquerra plus de crédibilité. 
En décidant de se situer en périphérie de l'orientation formelle, au contact de l'orientation non formelle et informelle, le conseiller va se placer au plus près du bénéficiaire en cherchant à faire parler au mieux chez celui-ci ses orientations non formelles et informelles, comme d'ailleurs le préconise le modèle du Life designing lorsqu'il recourt au récit biographique. Ce refus de se laisser enfermer dans l'orientation formelle, ce recours systématique aux deux autres formes d'orientation, constituent un atout de proximité bénéficiaire-conseiller dans cette immersion rendue possible au sein d'un espace de sociabilité qui valorise davantage le processus que les procédures, en décalage avec le modèle d'intervention ici étudié, qui reste davantage tributaire du procédural, même s'il invoque le processus.

Quoi qu'il advienne, le conseiller d'orientation ne saurait être assimilé de près ou de loin à un designer, sorte de démiurge qui prendrait une secrète revanche par rapport à la modestie du statut que l'institution lui octroie. Il doit au contraire ne pas rechigner à s'ouvrir à la dimension tragique de sa profession et de l'action qu'il mène. En cela, il ne représente pas dans l'ordre de l'humain une exception, car le tragique accompagne toute action humaine (Barel, 1987), que celle-ci consiste à s'orienter ou à conseiller dans l'orientation. Une telle dimension s'exprime dans le fait que toute action implique, pour rester humaine, d'être anticipée mais, paradoxalement, elle reste pour une part imprévisible dans sa réalisation ainsi que dans les conséquences qu'elle engendre et ce sans doute encore plus aujourd'hui en régime de complexité grandissante qu'hier. D'ailleurs, elle s'avère non seulement imprévisible mais également coûteuse : en effet les avantages qu'elle produit ne sauraient être dissociés des coûts parfois disproportionnés qu'elle engage. Mais ce défi tragique, celui de ne pas pouvoir réaliser ce que l'on veut ou ce que l'on entrevoit vaut la peine d'être couru, que l'on soit bénéficiaire ou conseiller; c'est la façon la plus authentique d'exister, aussi bien dans ses réussites que dans ses échecs, les premiers étant tragiquement inséparables des seconds, dans une sorte d'ambivalence irréductible. Vivre une telle ambivalence protège des déboires de vies adultes qui sous l'effet de l'une ou l'autre dépendance n'ont même pas eu l'opportunité de se poser la question de savoir où aller, à quelles conditions et comment exister. Ce sont de telles vies qui réclament urgemment l'aide d'un conseiller, muni du sens tragique qu'il donne à sa profession.

\section{Perspectives}

Revenons au titre du modèle qui nous préoccupe et qui semble faire un pied de nez à l'intention affichée dès le résumé initial rédigé par les co-auteurs. Or, tout lecteur le sait bien, peu importe le texte et son argumentaire, un papier n'existe que par son titre qui le personnalise; c'est donc pour finir ce dernier que nous allons interroger à travers cette grimace que le Life designing semble faire au constructionnisme social évoqué dès le résumé comme perspective choisie. Certes, nous n'avons pas la place ici d'épiloguer sur le déplacement épistémologique opéré, voire même la rupture lorsque l'on passe du constructivisme qui a déjà ses racines historiques et ses nombreuses lettres de noblesse, au constructionnisme, plus récent et au parfum postmoderne. $\mathrm{Ne}$ nous arrêtons pas au fait que les travaux constructionnistes se présentent dans la littérature, tantôt comme le prolongement du constructivisme, tantôt comme son contraste: alors que le constructivisme s'intéresse à la façon par laquelle le sujet se structure à travers ses expériences, le constructionisme, dans sa version contrastée, 
privilégie le mode d'élaboration que fait le sujet de la réalité qui l'environne. Constatons seulement que nous sommes là dans le face à face entre Life designing et constructionnisme social en présence de deux paradigmes opposés pour rendre compte du sujet s'orientant, le premier délibérément biologique, le second sociologique. À moins de vouloir ressusciter une variante de sociobiologie, on ne voit pas comment faire cohabiter ensemble ces deux paradigmes, pas plus que l'on imagine la place possible que pourrait occuper le psychologique, absent. La priorité doit-elle donc être donnée au premier paradigme, celui du titre, sur le second? Dans ce cas, accoler ensemble life et designing, c'est délibérément tourner le dos à toute forme de social pour se placer sous la protection d'une approche biologique, aujourd'hui très, voire trop en vogue dans sa version biomédicale. Une telle approche, mise en scène pour rendre compte du parcours de vie, ne peut être dissociée dans son contexte actuel, d'une double lecture posthumaniste et transhumaniste que le lecteur est susceptible de faire du life designing. Mais cela rejoint-il bien les intentions des auteurs?

- Une lecture posthumaniste peut être faite du modèle d'orientation qui nous est proposé dans la mesure où exister par soi-même dans son parcours professionnel à travers ses choix et orientations, c'est ici devoir se mettre en périphérie d'un dispositif technique qui va gouverner ses intentions, celui du conseiller et du modèle standardisé qu'il est amené à déployer, un modèle que l'actuelle mondialisation va rendre aisément généralisable, selon le vœu de ses auteurs, gommant par le fait même toute particularité culturelle et toute singularité individuelle; l'humain, comme d'ailleurs dans les autres systèmes techniques actuellement en vogue, en est réduit à jouer le rôle de périphérique au sein d'un système posthumain (Lafontaine, 2008) : il prend ou on le force à prendre rendez-vous chez le conseiller-spécialiste de construction de sa vie ou de gestion de son parcours, puis après, tout se déroule techniquement.

- Une autre lecture transhumaniste est suggérée par le recours au Life designing; ce dernier en effet dans ses capacités créatives évoque le Life Itself des courants transhumanistes (Rose, 2007), l'une et l'autre formulation renvoyant à l'idée de perfectibilité vitale, et par delà cette perfectibilité, à un refus de la finitude et à un déni de la mort; le Life designing peut alors être considéré comme une sorte d'élixir de jeunesse destiné à maintenir la vie, telle qu'elle s'exprime dans le parcours processionnel, professionnel ou pas, le plus longtemps possible fonctionnelle et donc de mettre en cause toute perspective de vieillissement; d'ailleurs le modèle ne fait jamais allusion à la question de l'âge, pas plus qu'il n'évoque celle des temporalités essentielles à toute orientation; la construction et le management de parcours sont atemporels, comme chaque cas unique est présenté de manière uniforme : pas de mouvement mais de l'éternité !

En fait, associer Life designing et constructionnisme social, n'est-ce pas faire ce que certains chercheurs nomment une biologisation des rapports sociaux (Cetina, 2005), heureux substitut postmoderne au projet d'émancipation sociale issu des Lumières, qui fut longtemps un phare tout en se trouvant aujourd'hui bien malmené. Ce n'est plus la société qu'il convient de changer, c'est désormais l'individu, jugé responsable de son orientation mais tributaire d'une responsabilité défaillante qu'il s'agit techniquement de conforter ; or derrière l'individu, client ou bénéficiaire, c'est la vie tout court, ce life itself qui devient un bien que le pouvoir biomédical entend gérer pour en maximiser son potentiel. 
Pourtant, il ne saurait y avoir d'orientation sans désorientation, ni réorientation, sans réussite ni échec et une telle orientation reste tributaire du contexte social dans lequel elle se déploie comme de l'avancée en âge de l'adulte concerné, jeune ou accompli, faisant que tous les possibles ne sont pas également possibles. Cette orientation, si modélisable soit-elle, et une modélisation bien tempérée constitue une aide très appréciable, relève de l'aménagement d'un espace singulier, une singularité porteuse plus d'universel problématique de la condition humaine que de mondialisé communicationnel. Mais cet espace singulier dans la façon par laquelle il se construit et se déconstruit au contact des vicissitudes du monde postmoderne relève plus d'une certaine idée de l'existentiel tragique propre aux risques associés à toute action, ici d'orientation, que de la perfectibilité humaine. Or le drame de l'orientation actuelle réside pour bon nombre de nos contemporains dans le fait qu'ils sont exclus de cet existentiel tragique, mis dans l'impossibilité de pouvoir y accéder. Pourtant, le tragique est incontournable car appartenant en propre à l'adulte agissant, alors que le drame fait d'une mésaventure qui tourne mal est, lui, évitable. Le modèle qui nous est ici proposé dans sa dimension préventive essaie de conjurer le drame. Y parviendra-t-il ?

\section{BIBLIOGRAPHIE}

- Barel, Y. (1987). La quête du sens. Paris : Seuil.

- Baubion-Broye, A. (éd.). (1998). Évènements de vie, transitions et construction de la personne, Toulouse : Érès.

- Bowman, L. C. (1979). The Argument from design. Sophia, 18, 1-13.

- Bridges, W. (2006). Transition de vie, comment s'adapter aux tourments de nos existences. Paris : Interéditions (original publié en 2004).

- Carré, P. (2005). L'apprenance. Paris : Dunod.

- Cetina, K. K. (2005). The rise of a culture of life. EMBO Report, 6, 76-80.

- Ciaravino, J. (2004). Un art paradoxal. Paris : L'Harmattan.

- Colaruso, C. A., \& Nemiroff, R. H. (1981). Adult development, a new dimension in psychodynamic theory and practice. New York : Plenum Press.

- Derrida, J. (1967). L'écriture et la différence. Paris : Seuil.

- Drevillon, J. (1966). L'orientation scolaire et professionnelle. Paris : Presses universitaires de France.

- Dubet, F. (1973). Pour une définition du mode d'adaptation des jeunes à travers la notion de projet. Revue française de sociologie, XIV, 221-241.

- Fayolle, C. (1998). Le design. Éditions Scala.

- Gergen, K. G. (1991). The Saturated Self. New York: Basic Books. 
- Gergen, K. G. (2001). Le constructionnisme social. Lonay : Delachaux et Niestlé (original publié en 1999).

- Ginzberg, E. (1951). Occupational Choice. New York: Columbia University.

- Lafontaine, C. (2008). La société postmortelle. Paris : Seuil.

- Milner, J.-C. (2006). Le juif de savoir. Paris : Grasset.

- Papanek, V. (1972). Design for the real world. New York: Panthron Books.

- Parsons, F. (1909). Choosing a vocation. Boston: Houghton-Mifflin.

- Piaget, J. (1967). Biologie et connaissance. Paris : Gallimard.

- Rose, N. (2007). The politics of life itself, biomedecine, power and subjectivity in the twenty-tirst century. New Jersey: Princeton University Press.

- Super, D. E. (1957). Psychology of Career. New-York: Harper.

- Vasari, G. (1550). Introduzzione alle tre arti del disegno (Trad. fr. : Paris, Les Belles Lettres, 2000).

\section{RÉSUMÉS}

Le présent article cherche à développer un point de vue critique sur le conseil en orientation à partir de la contribution originale intitulée Life Designing, co-écrite par neuf rédacteurs d'Europe et des États-Unis. Cette contribution est analysée dans ses points forts visant à élaborer un nouveau modèle de conseil en orientation à partir du concept de Designing, suite au constat fait d'une nouvelle organisation sociale du travail générée chez l'individu contemporain ; ce dernier en effet est de plus en plus souvent confronté à devoir assurer un parcours professionnel fait d'aléas, faute de s'en remettre à une trajectoire déjà prédéterminée, comme auparavant. Après avoir passé en revue les présuppositions du modèle qui en constituent les points forts, l'article se focalise sur un certain nombre de questions qu'il soumet à discussion, notamment celle de la mondialisation de l'orientation ici invoquée au regard des inévitables ancrages culturels, celle aussi de la signification à attribuer aujourd'hui au conseil en orientation, décliné tantôt dans sa forme activo-passive, tantôt dans sa forme pronominale, celle par ailleurs de la théorie de référence ici mobilisée et de la portée à lui conférer, le constructionnisme social, celle enfin du statut à conférer au professionnel de l'orientation, ce conseiller dont l'identité est bien malmenée : comment donc apprécier les perspectives offertes par le modèle du Life Designing en vue de refonder la professionnalité du conseiller en orientation?

The following article seeks to develop a critical view about counselling as it is described in the work entitled "Life Designing", co-written by nine European and American editors. This contribution is analyzed in its major points which aim at developing a new counselling model based on the ideas presented in "Designing" and emerging from observations of the new type of work organization, in all of its societal aspects, encountered by people today. People face more and more often the necessity to build their professional careers spontaneously because of a lack of predetermined trajectory as may have once existed. Having reviewed the premises of the model which constitutes its essential elements, the article focuses on a number of questions raised for discussion. First, the globalization of counselling brought out in relation to its unavoidable cultural moorings. Second, the meaning attributed today to counselling described either in its active form or in its reflexive form. A third question concerns social constructionism, the referential theory used here and the importance that should be given to it. And last, what status to offer the professional counsellor, whose identity has been placed in doubt. How do we 
understand the perspectives offered by the "Life Designing model" as we re-establish the professional character of the counsellor?

\section{INDEX}

Mots-clés : Conseil en orientation, Conseiller, Constructionnisme, Parcours de vie Keywords : Contructionism, Counselling, Counsellor, Life designing

\section{AUTEURS}

\section{JEAN-PIERRE BOUTINET}

Jean-Pierre Boutinet est professeur émérite en psychologie sociale à l'Université catholique de l'ouest, Angers, et membre de l'équipe de recherche CAFORE (IPSA/UCO Angers). Thèmes de recherche : conduites et cultures à projet, vie adulte et dispositifs d'accompagnement, environnements postmodernes et mutations des temporalités, l'âge de la responsabilité et ses nouvelles significations. Contact : UCO - 3 place André-Leroy - 49008 Angers. Courriel : jeanpierre.boutinet@wanadoo.fr

\section{CHRISTIAN HESLON}

Christian Heslon est maître de conférences en psychologie sociale à l'Université catholique de l'ouest, Angers, et membre de l'équipe de recherche CAFORE (IPSA/UCO Angers). Thèmes de recherche : psychologie des âges de la vie, conduites à projet, temporalités postmodernes.

Courriel : christian.heslon@wanadoo.fr 